\title{
Designing Interactive 3D Printed Models with Teachers of the Visually Impaired
}

\author{
Lei Shi \\ Cornell Tech, Cornell University \\ New York, NY, U.S.A. \\ ls776@cornell.edu \\ Zhuohao Zhang \\ Zhejiang University \\ Hangzhou, China \\ zhuohaozhang@zju.edu.cn
}

\author{
Holly Lawson \\ Portland State University \\ Portland, OR, U.S.A. \\ hlawson@pdx.edu \\ Shiri Azenkot \\ Cornell Tech, Cornell University \\ New York, NY, U.S.A. \\ shiri.azenkot@cornell.edu
}

\begin{abstract}
Students with visual impairments struggle to learn various concepts in the academic curriculum because diagrams, images, and other visual are not accessible to them. To address this, researchers have design interactive 3D printed models (I3Ms) that provide audio descriptions when a user touches components of a model. In prior work, I3Ms were designed on an ad hoc basis, and it is currently unknown what general guidelines produce effective I3M designs. To address this gap, we conducted two studies with Teachers of the Visually Impaired (TVIs). First, we led two design workshops with 35 TVIs, who modified sample models and added interactive elements to them. Second, we worked with three TVIs to design three I3Ms in an iterative instructional design process. At the end of this process, the TVIs used the I3Ms we designed to teach their students. We conclude that I3Ms should (1) have effective tactile features (e.g., distinctive patterns between components), (2) contain both auditory and visual content (e.g., explanatory animations), and (3) consider pedagogical methods (e.g., overview before details).
\end{abstract}

\section{CCS CONCEPTS}

\section{- Human-centered computing $\rightarrow$ Accessibility.}

Permission to make digital or hard copies of all or part of this work for personal or classroom use is granted without fee provided that copies are not made or distributed for profit or commercial advantage and that copies bear this notice and the full citation on the first page. Copyrights for components of this work owned by others than the author(s) must be honored. Abstracting with credit is permitted. To copy otherwise, or republish, to post on servers or to redistribute to lists, requires prior specific permission and/or a fee. Request permissions from permissions@acm.org. CHI 2019, May 4-9, 2019, Glasgow, Scotland UK

(C) 2019 Copyright held by the owner/author(s). Publication rights licensed to ACM.

ACM ISBN 978-1-4503-5970-2/19/05 ..\$15.00

https://doi.org/10.1145/3290605.3300427

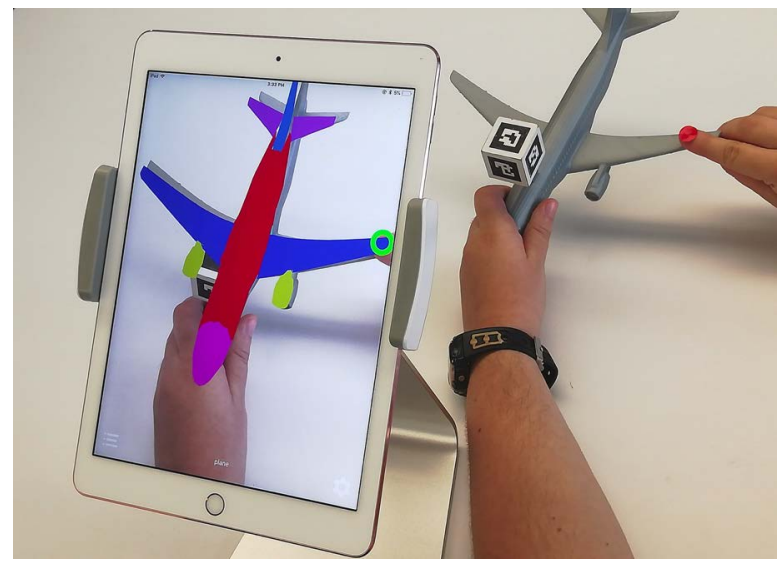

Figure 1: A visually impaired student uses a mobile application to learn about the Plane Model. The application speaks audio information when the student explores the model, and highlights different components on the model with colors accessible to low vision students. The iPad is on a stand so that the camera captures the student's interactions with the model.

\section{KEYWORDS}

Interactive 3D printed models, User-centered design

\section{ACM Reference Format:}

Lei Shi, Holly Lawson, Zhuohao Zhang, and Shiri Azenkot. 2019. Designing Interactive 3D Printed Models with Teachers of the Visually Impaired. In CHI Conference on Human Factors in Computing Systems Proceedings (CHI 2019), May 4-9, 2019, Glasgow, Scotland UK. ACM, New York, NY, USA, 14 pages. https://doi.org/10.1145/ 3290605.3300427

\section{INTRODUCTION}

Since students with visual impairments (VIs) can't access visual materials, 3D models are important learning aids. Teachers of the visually impaired (TVIs), who work with all students with VIs in the US in supplementary one-on-one or 
small group sessions, use 3D models to teach abstract concepts that cannot be conveyed by text alone. For example, a DNA strand is too small to present to a student with VIs, so TVIs may supplement a science curriculum by introducing a model to convey DNA's double helix structure [2]. These models help students better understand concepts, and improve their performance on exams [41].

Recent advances in 3D printing technologies has made 3D models more available. Previously, only a small number of expensive 3D tactile models were available for purchase $[2,3]$, so TVIs created their own models using arts and crafts materials $[41,53]$. With the help of consumer-grade 3D printers, TVIs now can design and print versatile 3D models [16]. In prior work, researchers and TVIs developed a variety of printed tactile learning materials for students with VIs [11, 30, 31, 34, 49].

However, 3D printed models are still limited compared to commercial models and visual materials used by sighted students. For example, a sighted student can learn geography and topology with a globe, using its text labels and colorcoded regions. Meanwhile, 3D printed models are typically monochrome, with no labels for a sighted or VI student to read. It is feasible to add braille labels to a 3D printed model, but there is limited space since braille takes up much more space than print. Researchers developed interactive 3D printed models (I3Ms), which could speak explanatory information to a student with VIs when he explores the model. Holloway et al. found that I3Ms provided a clearer tactile experience than traditional tactile materials [27].

However, most prior work focused on developing sensing techniques for I3Ms [18, 27, 39, 44-46, 48, 51]; little work has explored how to design and use I3Ms in an educational setting. Many questions remain before we can integrate I3Ms into educational practice. How should we design and modify the physical model of an I3M to meet teaching needs? What interactive elements (e.g., audio labels) should we add to an I3M? How would TVIs and their students use I3Ms? What tools would help support the design and use of I3Ms in a teaching session?

In this paper, we present two studies that investigate how to design I3Ms as effective teaching aids. In both studies, we worked with TVIs, domain experts who design tactile teaching aids for their students. In the first study, we led two design workshops with 35 TVIs, who modified 3D models we prepared, and added interactive elements to the models. They also explored existing I3Ms with an enabling desktop application (presented in our prior work [48]). Through the workshops, the TVIs provided suggestions about model modification (e.g., the size of physical models should be bigger), interactive elements (e.g., adding visual animations for low vision students), interaction techniques (e.g., supporting speech input), and pedagogical considerations (e.g., providing an overview before details), They also provided feedback for the existing I3M system. Despite the rich feedback gathered from a wide range of participants, the first study was limited to conceptual designs that were not implemented or used with students, and the TVIs were constrained by the models that we selected.

To complement the first study, we conducted the second study, where we collaborated with three TVIs individually to design and deploy sample I3Ms over seven weeks. First, we improved the prior system and developed a mobile application that supports the use of I3Ms in an educational setting. Then, we worked with the TVIs to design three I3Ms they chose: the Plane Model (Figure 1), the Volcano Model (Figure 11), and the Map Model (Figure 14). These I3Ms, which can be deployed using the mobile application, take gesture and speech commands as input, and support auditory (i.e., labels, detailed descriptions, audio effects) and visual feedback (i.e., animations, color highlights). At the end of the design process, the TVIs used and evaluated the I3Ms with their students. The students learned how to use I3Ms quickly and enjoyed the auditory and visual feedback. They understood the represented concepts better and demonstrated increased independence.

In summary, we contribute:

(1) Findings from two studies that revealed the opportunities, challenges, and design guidelines for I3Ms as teaching aids.

(2) Designs for sample I3Ms along with a mobile application ${ }^{1}$ that supports the use of I3Ms in an educational setting.

\section{RELATED WORK}

3D printing presents many opportunities for do-it-yourself solutions to accessibility problems. Researchers have applied this technology to create assistive devices like prostheses [26], hand grips [15], tactile interfaces [25]. With the help of a thriving maker culture and online communities, 3D printing is benefiting people with diverse needs [13, 29].

For people with VIs, 3D printing is a powerful educational tool $[14,16]$. Prior work focused on either designing teaching aids using regular, non-interactive $3 \mathrm{D}$ printed models, or developing different sensing and interaction techniques for I3Ms without deploying them with teachers and students. Our work bridges the gap between these two threads.

\section{Designing Printed Tactile Learning Materials}

Researchers and practitioners have prepared various printed tactile graphics and models for people with VIs. Grice et al.

\footnotetext{
${ }^{1}$ The source code of this application is available online at: http://InteractivePrintedModels.com
} 
converted Hubble Space Telescope images into tactile graphics [21]. Kolitsky 3D printed images from STEM disciplines like anatomy, along with embossed braille labels [32]. Gual et al. explored different designs of printed tactile maps [2224]. To the best of our knowledge, none of these materials, however, were designed with TVIs, nor were they evaluated by students with VIs.

Only a few projects involved students with VIs or teaching staff in their studies. McDonald et al. printed tactile aids to help students with VIs learn graphic design theory [34]. Kane et al. printed tactile graphics to show programming results to students with VIs in a computer science workshop [30]. Abigale et al. designed printed tactile books with teachers and caregivers of students with VIs [49, 50]. Jeeeun et al. further added movable components into printed tactile books, and evaluated their designs with TVIs [31]. Hu explored paradigms for 3D printed graphs with TVIs in a co-design process [28].

Prior work also contributed tools to lower access barriers to 3D printed learning materials. This is critical for enabling non-experts, like TVIs, to create and print their own 3D models. For example, Wedler et al. developed software that allows students with VIs to create and print chemistry models independently with minimal assistance from sighted co-workers [55]. VizTouch [11] software automatically generates tactile graphics of mathematical graphs. Taylor et al. developed a web tool that allows people with VIs to generate 3D map models with specified locations [52]. Similarly, Götzelmann et al. created a pipeline that could convert online map data into printable models [20].

Compared to these projects, our work involved both TVIs and students with VIs, and we specifically explored how to design I3Ms, which are proven to be more effective than regular prints [27], as teaching aids.

\section{Interactive 3D Printed Models and Maps}

To make 3D printed models more powerful, researchers contributed different techniques to add interactivity to these models. Most of these techniques involved conductive materials, additional electronics, and new printers. Schmitz et al. [43], Taylor et al. [51], and Götzelmann [18, 19] printed models with conductive parts and put them on a touchscreen. When a user touches the conductive parts, the touchscreen recognizes the gesture and plays an audio label. Since the models must be in contact with a touchscreen, a user cannot explore the models freely in the air, and the designs of these models were usually limited to maps. In addition to touchscreens, prior work also used Arduino controllers [27, 33] and electrodes [56] to sense users' inputs on conductive models. Researchers also explored new printers that can print electronic components directly inside models [36,37]. These approaches require TVIs and students with VIs to obtain electronics and new printers.

In prior work, we used acoustic sensing to make 3D prints interactive $[44,46]$. We designed models with printable percussive components, which can generate unique sounds when flicked. A mobile application recognizes the sounds and speaks audio labels. This method does not rely on embedded electronics. However, the precision of acoustic sensing was limited, and adding more audio labels would likely decreased the accuracy.

More recently, we used cameras to enable interactivity in 3D models [48]. Markit is a design tool that allows a user (e.g., a TVI) to add annotations on a 3D model. Talkit is a desktop application that allows a user with VIs to explore the annotations on the model. Using the webcam from a laptop, Talkit locates a model by sensing the tracker mounted on the model, and finds the user's finger by tracking a red sticker (see Figure 4 and Figure 5). When a user puts his finger on an interactive element, Talkit speaks the name of the element. The user can say "more" to hear a detailed description of the element. Similar to other camera-based systems, the user must keep the model and their finger within the field of view of the camera. Both Talkit and Markit were evaluated in lab studies. We used Talkit in our first study, and developed a new mobile application with additional features (e.g., visual effects) for our second study.

Most prior work focused on interactive sensing techniques in controlled lab settings with adults with VIs; only a few projects have been designed and evaluated with students with VIs in real teaching environments. Giraud et al. [17] designed one interactive map in collaboration with a specialized teacher, and evaluated it with 24 students with VIs in controlled lab studies. Brule et al. [12] designed a multisensory map and conducted a field study with children and caretakers in geography classes. Although maps are important teaching aids, we focus on designing I3Ms for a variety of concepts and curricular areas. Our work extends the findings from these studies, and provides design guidelines and sample I3Ms that can be used in educational settings.

\section{WORKSHOP STUDY}

The primary goal of the first study was to understand how we should design I3Ms as teaching aids, and derive design guidelines for future model designers. We also aimed to solicit feedback about existing I3Ms so we could improve the technologies to better support the use of I3Ms in classrooms.

We chose to achieve our study goals through design workshops. A workshop setting allows the researchers to engage with a relatively large number of participants in a short amount of time, allowing some one-on-one conversation as well as discussion among the participants. 
Table 1: Different groups and their chosen models in the workshop study

\begin{tabular}{|c|c|c|c|c|c|c|c|c|}
\hline Group Number & 1 & 2 & 3 & 4 & 5 & 6 & 7 & 8 \\
\hline Chosen Model & Elephant & Heart & Map & Skull & House & Heart & Elephant & Map \\
\hline
\end{tabular}

\begin{abstract}
Method
Participants. We recruited participants for two workshops, which were conducted in two state conferences of the Association for Education and Rehabilitation of the Blind and Visually Impaired (AERBVI), an organization for professionals who provide services to people with VIs [1]. The two workshops followed a same protocol but were hosted in different locations with different participants.

Sixteen TVIs (3 males, 13 females) participated at the first workshop, whose reported years of experience ranged from 3 to 40 years $($ mean $=21, \mathrm{SD}=13)$. One identified as low vision, two identified as blind, and 13 identified as sighted. Nineteen TVIs (5 males, 14 females) joined the second workshop. Their reported years of experience ranged from 2 to 41 years $($ mean $=17, \mathrm{SD}=12)$. One identified as low vision, one identified as blind, and 17 identified as sighted.
\end{abstract}

Apparatus and Materials. We printed seven 3D models from Thingiverse.com. When exploring possible models from online resources, the researchers on the team, including one who is an expert in education for students with visual impairments, identified models that would be likely be used by TVIs in educational settings. The models we chose were (1) a map of the U.S. (Thing: 61210), (2) a rocket (Thing: 100070), (3) an anatomical heart (Thing: 932606), (4) an elephant (Thing: 182136, as shown in Figure 2), (5) a house (Thing: 270223), (6) a skull (Thing: 622390, as shown in Figure 3), and (7) a DNA (Thing: 1281735). We used Makerbot and Ultimaker printers to print these models with PLA filament. To allow participants to document modifications and annotations on the printed models, we prepared Play-Doh, stickers, sticky notes, pens, markers, and poster boards.

To allow participants to experience I3Ms with existing annotations, we created three I3Ms and ran Talkit on three Macbook laptops. The three I3Ms were: Cell, Globe, Map, models used in prior work [48].

Procedure. Each workshop lasted 1.5 hours. We began each workshop with a short presentation introducing 3D printing technologies, I3Ms, and the design of Talkit. Then, participants split into groups (G1 to G4 in the first workshop, and G5 to G8 in the second workshop). Each group was asked to design one I3M.

At the start of their design process, each group chose one model from the seven models we printed. The participants chose models based on their interests, and on a first-come, first-serve basis. Their choices are shown in Table 1.
Then, each group modified the model using materials like Play-Doh, and used markers and stickers to design interactive elements on the model. They brainstormed the type and content for these elements, and how students would interact with them. During the design process, they also explored our existing I3Ms using Talkit.

At the end of the workshops, each group delegated one or two representatives to briefly present their designs.

Analysis. We video recorded the presentations and took photos of the modified models. Two researchers developed themes from the recording transcripts using axial coding [42]. We refer to members of each group by their group number (G1-G8).

\section{Findings}

Model Modification. Participants provided ways to improve and modify the designs of 3D printed models.

Size: Four groups shared concerns about the printed models being too small and explained that their size could impede tactile exploration and understanding of the concepts related to the referent object. G1 and G7, who both chose the elephant model, thought the small model would mislead students. G1 said it would be better if they could "have something accompanying that model to show scale." For example, a 3D print of the foot of an elephant. Similarly, G4 also wanted to have an anatomically correct skull to show the correct size of the skull. G8 wanted a larger map model so students could feel each of the states.

Texture: Participants were unsatisfied with the limited printing quality we had and the solid printing materials we used. For example, the printing imperfections resulted in ridges on the top of the elephant model (see Figure 2) and "stringy" surface on the heart model. G6, who chose the heart model, said the model "should be lovely to touch" instead of being "like rough sandpaper." They wanted to use a rubbery material to make the texture of the model more similar to that of a heart. G1 thought that "different parts [should have] different texture." They also suggested printing the model in a higher resolution to avoid imperfections.

Tactile Differentiation: G2, G4, and G8 wanted to add tactile patterns on the models to make different components more distinct from one another. G4 said "there wasn't much tactile discrimination between them (different components on the skull model)," and they used Play-Doh to indicate different 
tactile patterns they would add onto the model, as shown in Figure 3. G2 and G8 had similar thoughts on the heart model and the map model.

Visual Cues: Four groups wanted to add visual cues to their models for low vision students. G7 thought the elephant model should be in the color of an elephant instead of being black, because most students with VIs have usable vision. Both G2 and G6 wanted to make a multi-color heart model. For example, G2 thought the arteries and veins should be color-coded to demonstrate blood flow. G4, who indicated that tactile patterns were useful to blind students, thought higher contrast would help low vision students better understand the model.

Removable Components: Two groups were interested in removable components, which would allow students to explore the relationships between different components. For example, G5, who chose the house model, wanted to add a removable roof, so students could explore the inside of the house when taking off the roof. Similarly, G4 wanted to create a removable brain component inside the skull model.

Interactive Elements. After modifying the physical models, the participants designed interactive elements for their models. In addition to audio labels and detailed description that were widely deployed in prior work [18, 27, 39, 44-47], participants also used audio effects and animations.

Labels and Description: In general, the participants were all in favor of annotating model components with name labels, which could be followed by detailed descriptions when queried by users with VIs. They labeled each component of their models, and the number of labeled elements ranged from four (on the skull model by G4) to 12 (on the heart model by G6). They also added detailed descriptions about each component's function (e.g., the function of arteries), size (e.g., the size of an elephant leg), texture (e.g., the texture of elephant skin), related metrics (e.g., the population of a city), and surrounding components (e.g., surrounding states). G1 wanted to add a story to the elephant model in addition to a factual description.

Audio Effects: Six groups used audio effects in their interactive elements. Both G1 and G7 wanted their I3Ms to play elephant sounds when a student touched the trunk on the elephant model. G2 wanted the sound of the heartbeat. G6 associated heartbeat sounds with gestures, suggesting that "two taps (tapping on the model twice) would [play] like an active heartbeat, uh, maybe three taps would be like a very active heartbeat." For the map model, G3 added state songs and the sound of a state bird, and G8 wanted to have the sound of a state animal.
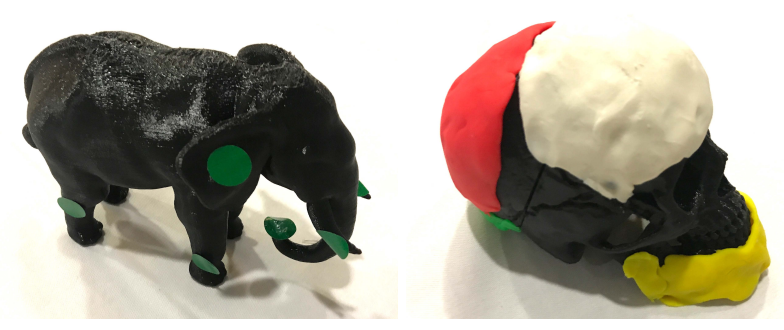

Figure 2: Participants modi- Figure 3: The participants fied the elephant model, us- modified the skull model. ing stickers to indicate loca- They used Play-Doh to intions that triggered annota- dicate different tactile pattions. Note the printing im- terns on four components. perfections at the top of the model, which participants found misleading.

Animations: In addition to audio and visual feedback, G2 wanted an animation of a beating heart on the heart model.

Interaction Techniques. Participants suggested different input and output techniques for the interactive information on the models.

Input Techniques: Gestures were the most common input technique suggested. For example, performing single and multiple taps on a model (G1-G6, G8) to get audio labels and detailed descriptions, respectively. G4 designed buttons near their model, with which a student could retrieve and record audio annotations. In addition, some participants wanted speech input. For example, G3 wanted simple commands like "Song" to activate audio effects on the map model. They also mentioned more conversational commands like "What's this state's song?" Overall, these input techniques are similar to what we found in our prior work [47], where we designed input techniques directly with adults with VIs.

Output Techniques: In addition to auditory feedback (e.g., annotations and audio effects) and visual feedback (e.g., animations) commonly mentioned by participants, G1, G4, and G6 also wanted to connect refreshable braille displays to the I3Ms as one potential output channel. The display could provide longer descriptions that students could access in braille. For example, G6 wanted to use a braille display to describe the complicated structure of the heart model.

Pedagogical Considerations. When describing how they would use I3Ms in their lessons, participants provided four suggestions.

Overview of a Model: The participants pointed out that students should have an overview of a model before going into more complicated details. G3 said that some students focused on details and missed the overview when studying a 
map model. G4 thought that students should know "the total number of things to find" on the skull model. The overview could be provided by a TVI or through the I3M.

Changeable Digital Content: The participants wanted to reuse a same printed model with different digital content. For example, G4 wanted to "erase that information (interactive elements) and use it with a different student." They also suggested changing the content on a model as a student advances in their studies.

Connections between Models and Referent Objects: As discussed previously, most 3D printed models had a limited size and a low-fidelity texture, which could lead to a misunderstanding of their referent objects. The participants wanted their students to be aware of the difference between a model and its referent object, and provide different solutions. For example, G4 wanted to bring a real skull along with the model.

Student-Made Annotations: G4 wanted to allow students to record their own annotations. They said, "if he tapped the button or double tapped, he could add his research to that." They thought that these annotations could be used in presentations or a science fair.

Feedback for the System. The participants also provided feedback for the Talkit application and the design of the physical trackers.

Application Setup: Some participants tried the Talkit application, and felt the setup of the desktop application was inconvenient. For example, the current camera position might lead to a high cognitive load for students with VIs. As shown in Figure 4, the Talkit application used the webcam of a laptop to track a model and the user's gestures. In this situation, as G3 said, "the kid has to reorient themselves when they put the map in front of them... and because so many of our kids also have perceptual issues, that might be tough." They suggested setting the camera above the user or right in front of them, facing the same direction as the student (FIgure 5).

Physical Trackers: A few participants discussed the disadvantages of the $3 \mathrm{D}$ tracker, which is a $2 \times 2 \times 2 \mathrm{~cm}^{3}$ cube covered with five fiducial tags. The tracker enabled the Talkit application to locate the position of the model. However, the tracker could feel bulky on some models.

\section{Discussion and Forethought}

The goal of this study was to derive design guidelines for I3Ms from TVIs. Thirty-five TVIs participated in this study, and provided suggestions about the designs of I3Ms from different aspects, such as model modification, interactive elements, interaction techniques, and pedagogical considerations. Although we collected data from a wide range of TVIs

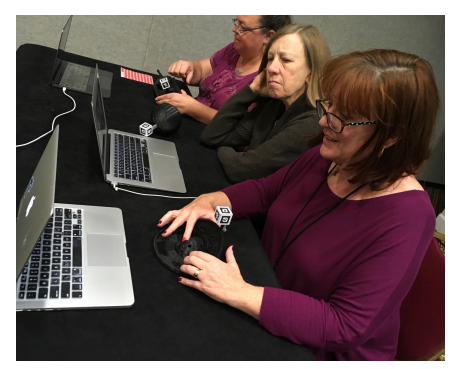

Figure 4: Three participants tried existing I3Ms with the Talkit application running on a Macbook. The application uses the webcam of the Macbook to detect where the user is touching the model.

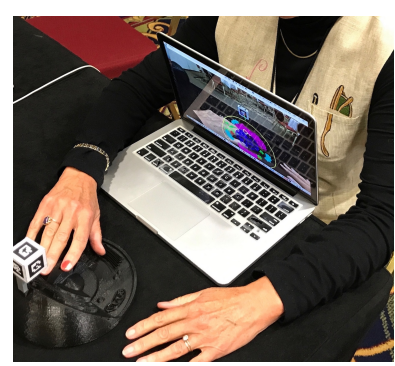

Figure 5: Unsatisfied with the orientation settings in Figure 4, a participant rotated the laptop so that its camera faces the same direction as the user. during the workshops, the data was mostly about conceptual designs that were not implemented or used with students. Moreover, the TVIs were constrained by the seven 3D prints that we provided as a foundation. The fact that participants were not able to choose models from a larger set was one limitation in the first study. We wanted to gather more comprehensive findings by deploying I3Ms with TVIs in their classrooms. Thus, we conducted another study, where we worked with three TVIs to design and deploy I3Ms. We will discuss the design guidelines of I3Ms with the findings from both studies at the end of this paper.

The first study also exposed some technical limitations of the Talkit application, which needed to be addressed before deploying I3Ms in classrooms. First, the application should support visual content. Animations and visual cues are useful for low vision students. Second, the participants showed interests in audio effects (e.g., the sound of a heartbeat), while the current application only spoke descriptive texts. Third, the setup of the application should be easier. The participants pointed out that the current setup would lead to high cognitive load for students. Last but not least, the application should support other forms of trackers (e.g., 2D fiducial tags). To address these limitations, we improved the application, which we describe subsequently.

\section{INSTRUCTIONAL DESIGN STUDY}

The goal of the Instructional Design study was to explore the design of I3Ms in practice. While the Workshop study aggregated suggestions from conceptual designs, this study allowed us to work with individual TVIs in-depth, and deploy I3Ms in their classrooms. Both studies answered our research question: how should we design I3Ms as effective teaching aids? In this study, we specifically wanted to explore (1) How would TVIs design and use I3Ms in their lessons? (2) 
Are the I3Ms designed by TVIs useful and effective in the classrooms?

Instructional design (ID) is the practice of developing learning experiences [35]. The development of technology has shaped the theories and guidelines of ID over time [40]. In our case, ID has two components: the design of I3Ms and the design of teaching plans. We worked with TVIs to develop and test these two components over seven weeks. Through this process, we designed three I3Ms along with teaching plans, and gained feedback from TVIs and their VI students.

\section{Method}

Apparatus: Talkit++. To deploy I3Ms in TVIs' classrooms, we developed a mobile application, Talkit++, as shown in Figure 6. To deploy I3Ms in TVIs' classrooms, we developed a mobile application, Talkit++, as shown in Figure 6. When designing the app, we incorporated TVIs' feedback gathered during the first study, and the suggestions from blind adults obtained on similar applications in prior work [48].

Like Talkit, Talkit++ tracks finger gestures using a red sticker, locates a model using a physical tracker, and provides audio labels and detailed description. The application also recognizes simple speech commands like "more." In addition, we added new features:

- Visual Cues: The application visually highlights the components on a model (see Figure 1).

- Animations: Users can trigger animations using a button on the touchscreen interface (see Figure 12).

- Audio Effects: Designers can add audio effects on I3Ms.

- 2D Trackers: In addition to 3D trackers, Talkit++ also supports tracking models using 2D trackers, which are less intrusive, as shown in Figure 11 and Figure 14.

- iOS Compatibility: The application runs on iOS devices.

Talkit++ was developed in Swift and $\mathrm{C}++$. We cross compiled computer vision libraries like Chilitags [9] and OpenCV [10], and called them in Swift. Talkit++ uses SpeechRecognizer [6] to detect speech commands. As for output, Talkit++ uses a text-to-speech engine [5], plays audio effects through AudioPlayer [4], and displays animations using UIImage [7]. We also updated Markit, the I3M design tool, to work with the new features.

To use an I3M, a user with VIs must put a red sticker on his fingernail, and run Talkit++ on an iOS device (e.g., an iPhone or an iPad). The user can put the device on a stand to adjust its camera position (see Figure 7). In the current application, the student needs to select the digital content that works with the model using a button on the application. In the future, users could select models using speech input. Once selected, the model speaks an overview of the model. Then, the user can explore the printed model tactilely. When his finger touches an interactive element, the model plays

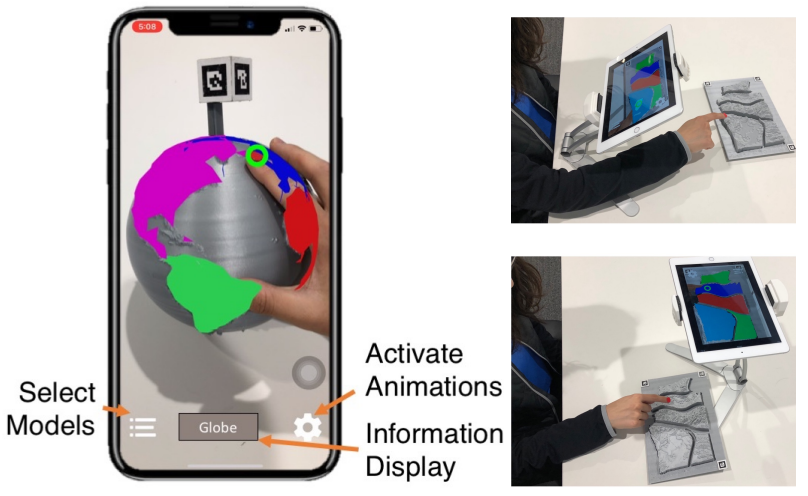

Figure 6: The user interface of Figure 7: Two ways the Talkit++ application, which a user could posiis accessible with the built-in tion an iPad using a VoiceOver screen reader. stand.

audio labels and audio effects. He can also say "more" to get a more detailed description.

Participants. Three TVI participants, denoted as T1 - T3, joined our study. All participants were female, and their ages were 52 (T1), 34 (T2), and 24 (T3). Their years of experience as TVIs were four (T1), one (T2), and one (T3). We recruited them through a Master's level personnel training program for TVIs, but they were already teaching students with VIs. In total, ten students participated. Instead of reporting the information of each student, we report the aggregated demographic data of the students to preserve their privacy:

- T1 planned to work with six students, whose ages were: eight, nine, nine, nine, 10, and 11. Four of them were blind, and the other two were low vision. Three of them had learning disabilities.

- T2 planned to work with three students, whose ages were: 16,18 , and 19 . Two of them were blind, and the other one is low vision. All of them had had learning disabilities and/or attention-deficit/hyperactivity disorder (ADHD).

- T3 planned to work with one blind student, whose age was 11 . The student did not have other disabilities.

Procedure. The entire study lasted seven weeks, and we followed ADDIE [38], a commonly used process in ID, to develop I3Ms. ADDIE is an acronym for five phases: Analysis, Design, Development, Implementation, and Evaluation. A researcher, who was not co-located with the participants, scheduled weekly 30-minute remote meetings with each TVI. In some cases (e.g., design iteration), they had extra short remote meetings. Besides the meetings, they communicated via emails. The researcher spent around 12 hours in total meeting with the three TVIs during the study. 
Week 1: Analysis. The goal was to (1) introduce the research project to the TVIs and (2) analyze and articulate the teaching needs. The researcher introduced I3Ms, and demonstrated the capabilities and limitations of I3Ms. Then, the TVIs were asked to think about who they would teach and potential concepts they wanted to teach with I3Ms. After the meeting, the TVIs were instructed to search online and come up with three potential teaching concepts.

Weeks 2-3: Design. The goal was to (1) design a physical model for each TVI, and (2) add interactive elements for the model. First, the researcher worked with the each TVI to choose one teaching concept and design a corresponding model. They chose a concept based on design complexity, printing feasibility, and feedback from peers. Then, the researcher helped the TVIs design their selected models. With the researcher's encouragement, the TVIs used digital models, drawings, and diagrams to illustrate their ideas.

After designing the physical models, the TVIs designed interactive elements. They were asked to provide the textual content of the overview introduction, audio labels, and detailed descriptions. Optionally, they also added audio effects and animations. They provided multimedia materials (e.g., audio files, YouTube videos) to the researcher if they wanted these features incorporated in their I3Ms.

Week 4: Development. The goal was to develop I3Ms and teaching plans. The researcher modified models found online to meet the TVIs' designs and printed them out. When the researcher encountered design or printing issues, he discussed these problems with the TVIs and together they adjusted the models.

Then, the researcher added textual content, audio files, and animations to the models. The audio files and animations were created from the materials that participants provided. The TVIs did not use 3D modeling software themselves during the study; the researcher iterated on the designs with feedback from the TVIs multiple times during the study.

During this phase, the TVIs were asked to develop their teaching plans for using I3Ms in their lessons.

Weeks 5-7: Implementation and Evaluation. The researcher and collaborating personnel university program provided research materials to the TVI participants. For each TVI, we prepared an iPad with the Talkit++ application, an iPad stand, finger stickers, trackers, and the selected printed model. An accessibility specialist and other researchers tested the usability of the application before delivering it to the TVIs. The TVIs had at least two weeks to get accustomed to the application before handing it to their students.
The TVIs implemented their teaching plans with their students. After each teaching session, the researcher interviewed TVIs. In the last lesson with I3Ms, the TVIs asked their students to provide feedback on the I3Ms.

Data Collection. We recorded all the remote meetings between the researcher and each TVI, and the TVIs provided their students' feedback on I3Ms during the meetings. We only used the data provided by the TVIs because of feasibility and privacy concerns. Two researchers transcribed and coded the recordings of the researcher's meetings with the TVIs.

\section{Design Deliverables}

Initial Concepts and Model Selection. The participants chose models according to their teaching curricula and students' individual capabilities. Initially, each of them proposed three models and narrowed down their choices based on feedback from their TVI colleagues, braillists, and the researchers.

T1 wanted to teach the structure of the earth, the structure of a flower, or the structure of a volcano. A model of a flower was too fragile to print. After conferring with her classmates, she decided to design an interactive volcano model, which would fit her teaching agenda and be more suitable for her students.

T2 wanted to teach the concept of an airplane, or animals like birds and elephants. After discussing with her peers and students, she chose an airplane model.

T3 initially proposed a model for conducting electricity experiments. She wanted to let students assemble printed components (e.g., a printed battery and a printed bulb) together and then get interactive feedback. This idea required interactions between 3D prints, which was not well supported by the current Talkit++ application. Besides the experiments, T3 was also interested in teaching with an airplane model or a printed map. We chose a map model to avoid duplicates.

Model Iteration. The TVIs iterated on the designs of their chosen models with the help from the researcher.

The Volcano Model: T1 wanted the external tactile textures of a mountain with the internal structure of a volcano. The researcher proposed an initial design by combining Thing:1316498 and Thing:1370593, as shown in Figure 8. However, T1 thought the model was too complicated. Thus, we iterated on the design and replaced the internal structure with the design from Thing: 1290606. In the final design, we made the model simpler. Since all the components are on one 2D surface, we used 2D trackers. We added two in case one of them was blocked by a students' hand. The dimensions of the model are $22 \times 15 \times 13 \mathrm{~cm}^{3}$.

The Plane Model: We modified Thing:182252, as shown in Figure 9 . The dimensions of the model are $23 \times 21 \times 6 \mathrm{~cm}^{3}$. The 


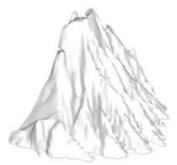

Side View

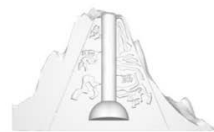

Initial Design

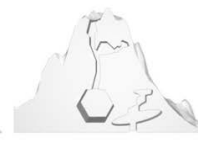

Iterated Design

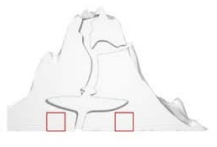

Final Design
Figure 8: Different designs of the Volcano Model. The final design includes two $2 \mathrm{D}$ trackers, which are highlighted in red. The model is designed for T1's students.

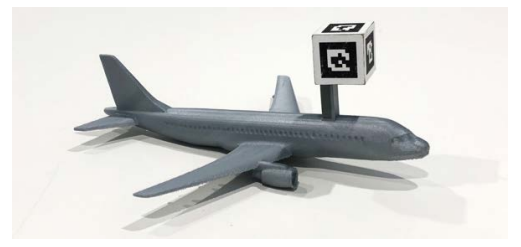

Figure 9: The Plane Model designed for T2's students.
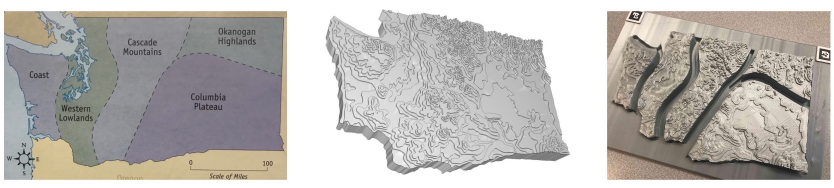

Figure 10: The materials and final design of the Map Model designed for T3's students.

application could still locate the 3D tracker when students rotate the model. T2 was really excited about the model, and said "This is something our students really could not see or experience in any other way."

The Map Model: T3 provided a diagram of a Washington State regional map, as shown in Figure 10, to the researcher for reference. The researcher found a model with similar content from GrabCAD. T3 wanted separate components for each region. The researcher iterated on the design of the model with T3, and designed a map puzzle. As shown in Figure 10, the puzzle consists of five pieces and a base, which has three 2D trackers on its corner. A student could take the pieces off the base. The dimensions of the model are $26 \times 17 \times 4 \mathrm{~cm}^{3}$.

Interactive Elements. The researcher helped the TVIs design and add interactive elements.

The Volcano Model: T1 designed audio labels and detailed descriptions for four components, as shown in Figure 11. In addition, she wanted an animation of a volcano eruption. The researcher designed an animation based on a YouTube video she provided, as shown in Figure 12. The animation also came with an audio introduction, and could be triggered by touching a button on the iPad screen.

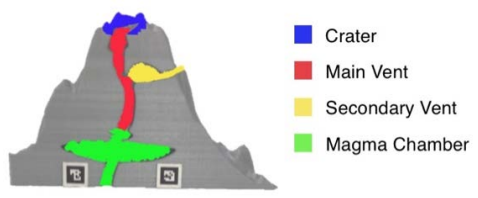

Figure 11: The interactive elements on the Volcano Model.
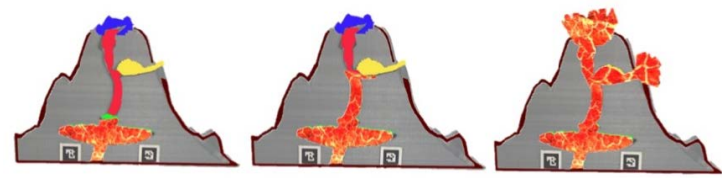

Figure 12: The animation of the Volcano Model.

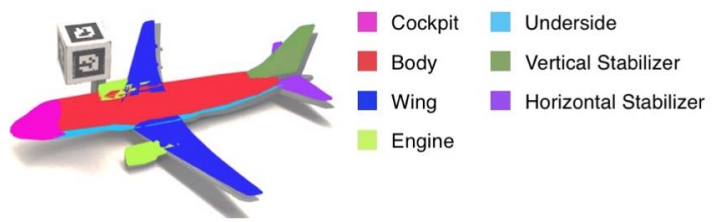

Figure 13: The interactive elements on the Plane Model.
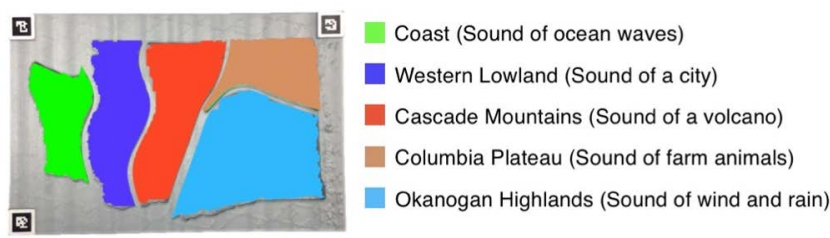

Figure 14: The interactive elements on the Map Model.

The Plane Model: T2 designed audio labels and detailed descriptions for seven components, as shown in Figure 13. She also designed three audio effects: (1) a recording of a pilot speaking for the Cockpit component, (2) the sound of people chatting for the Body component, and (3) the sound of an engine propeller roaring for the Jet Engine component. The audio effects played after the audio labels, and the detailed descriptions could be retrieved by saying "more."

The Map Model: T3 designed audio labels, detailed description, and audio effects, see Figure 14.

Teaching Plans. The TVIs developed teaching plans that incorporated the models.

The Volcano Model: T1 planned to have two group sessions with her students. In the first session, she planned to introduce basic knowledge about volcanoes (e.g., what a volcano is and how it works). Then, she wanted to let her students explore the physical model and use the I3M to learn the 
detailed structure of a volcano. In the second session, she developed assessment prompts to have the students recall different components of the model and assess learning using I3Ms. In the end, she planned to evaluate the I3M with her students.

The Plane Model: T2 planned to have two group sessions with her students. Her teaching plan followed a similar structure to T1's plan.

The Map Model: T3 wanted to have three sessions with her students. In the first session, she wanted to introduce the basic information about Washington State and its five regions. Then, she planned to have the students experience the physical model without any interactive features. In the second session, she planned to help her student set up and use the mobile application. In the third session, she wanted to let the student set up the application independently. She also wanted to assess her students and evaluate the I3M, similar to T1's plan.

\section{Findings}

In general, all the TVIs thought the sessions were successful, and their students were excited about the I3Ms. T3 said "she (her student) was really, really excited about it and she thought it was really cool." We asked the TVIs to define "success" in terms of system performance, the design of interactive elements, the progress of their students, and students' feedback.

System Performance. In general, the Talkit++ application performed well in the study. T3 said, "the actual technology, it was recognizing very quickly what she was touching." However, some students encountered usability issues.

Four out of six students working with T1 had difficulty activating interactive elements. Two students were not sure that "the sticker had to face the iPad," and stick their fingers in the holes. The application couldn't recognize their fingers without a visible red sticker. T1 thought it might be helpful to make a sticker that "goes all the way around their fingers." Another student, who wore nail polish, had to fold her other fingers because the system misclassified the fingernail polish as a sticker. One student moved his finger around the place quickly, and the application could not detect his moving fingers. Reflecting on the all sessions, T1 said most students managed to use to the system in the end.

T2 had a "very dependent" student who needed a lot of guidance to find the different parts of the model. She ended up providing hand-over-hand assistance.

T3's student kept moving the model away from the application's field of view. She said that "the student kind of gradually would shift the model around... The iPad would maybe stop recognizing." T3 ended up using her laptop to set boundaries for the student.

Interactive Elements. The TVIs thought their students enjoyed both the auditory and visual elements, but wanted more content.

All the TVIs liked the implemented auditory feedback. T2 thought the audio effects were unique and said "the sound of the people talking and the motors and the pilots inside the cockpit. Those are some elements that we couldn't produce otherwise." T1 thought it would be better if the I3M had more information, because she thought her students "got bored kind of fast." She suggested additional layers of information and more detailed explanations.

T1, the only TVI that had an animation on her model, thought the animation was attractive and effective. She taught the I3M in a group session, and she asked her students to try the model in turns. She found one low vision student was attracted to the animation, and "kept sticking her head in the way when the first girl was using it." Even for blind students, the animation was still useful, because "it's still interesting to listen to [the audio of the animation]."

Learning with I3Ms. The students became familiar with I3Ms quickly and used them independently. For example, T1 said her students learned how to use the models in her first session quickly, and said "they didn't need more than a few seconds of instruction to do it." In her second session, she let her students explore the model independently, and "they all remembered how to use it." Some students were even able to set up the I3Ms by themselves. T3, who tried to let her student launch Talkit++ and put a sticker on by herself, was surprised that the student finished the setup quickly. She said excitedly, "Wow, a fourth grader could use this independently after a couple of times practicing with a teacher." She attributed this to the accessible design of the application, which allowed the student to use VoiceOver, the iOS built-in accessibility screen reader. T3 thought, with practice, the student should be able to setup the stand for an iPad and position the model independently.

I3Ms afforded a unique, advanced learning experience with tactile, auditory, and visual feedback. For example, T1 said, "for my kids who can't see at all, they wouldn't be able to understand a diagram of the inside of a volcano. So it helped them to be able to feel what the outside is like, and the inside parts." T2 liked the design of the Plane Model, and said "Neither of them had been inside an airplane before ... using the 3D model, it was a good way to give them a concept of the shape of an airplane and how it works." All three TVIs reported that their students understood the taught concept better after using the I3Ms. T3 said her student knew all the names and descriptions of the five regions on the Map Model in their final assessment. 
Students' Feedback. The TVIs asked the students about their feedback on I3Ms.

All students liked I3Ms and thought the model helped them understand the taught concepts. They felt the models were better than traditional tactile graphics and wanted to use I3Ms again in the future.

When asked what other concepts they wanted to learn using I3Ms, the students showed a wide range of interests. The student working with T3, aged 11, was interested in a "Tactile Town," where the application could name shops like Starbucks. The students who learned the Plane Model were interested in cars and motorcycles. Among the students working with $\mathrm{T} 1$, one was interested in learning "how rain comes down," while the others were interested in planets, the galaxy, a space shuttle, and a model of Hawaii.

The students were asked to compare using I3Ms with teacher instruction only. Four students explicitly said they would prefer learning with I3Ms independently. T3's student said, with the help of I3Ms, she wouldn't have to take someone's time and become more independent. which was echoed by one of T1's students. The other two students thought the auditory and visual feedback, which were designed by TVIs, were more intriguing compared to a TVI.

\section{DISCUSSION}

We conducted two studies to explore how to design I3Ms as effective teaching aids. The first study involved more participants but only explored the designs conceptually, while the second study explored the research question through an in-depth design process with fewer participants. Both studies shed light on the design of I3Ms. We discuss the findings and implications from both studies below.

\section{Pros and Cons of I3Ms}

In the first study, the TVIs were interested in using I3Ms but were concerned about the quality of the models and the usability of the system. In the second study, after using the improved system with collaboratively-designed models, the three TVIs were satisfied with the technology but wanted to have more interactive elements. For example, T1 thought her students browsed through the content quickly, and wanted additional instructional material in her model. This suggested that our iteration on the design of the initial prototype addressed the issues raised in the first study.

Through the TVIs, we gained insight into the students' reaction to I3Ms. Of course, this was not a controlled experiment; we did not evaluate the students' learning progress with I3Ms versus another learning method. However, the qualitative data from the TVIs provided positive initial feedback about the students' experiences. According to the TVIs, their students were intrigued by the I3Ms and learned how to use them quickly. More importantly, they could learn concepts independently. Some students were even able to set up their I3M by themselves.

Our studies showed that I3Ms provided a customizable and interactive learning experience. With I3Ms, TVIs can design models and content to meet their plans, and students can gain a lot of knowledge from auditory and visual information, which is not available through traditional teaching aids (e.g., tactile graphics). In prior studies, I3Ms were also proven to be capable of facilitating space and text memorization [17] and providing an overview understanding of concepts [27].

With current consumer-grade 3D printing technologies, it is important to note that I3Ms still have limitations in printing quality and model designs. Most printed models have printing imperfections, and will not feel the same as the referent objects. In addition, it still takes a nontrivial amount of time to design, print, and prepare annotations for models. As printing technology improves in the coming years, I3Ms will be easier to produce and-we hope-more widely deployed.

\section{The Roles of TVIs}

The participation of TVIs is important in the design process of I3Ms because they understand the diverse needs of their students. In our study, the TVIs customized the content of I3Ms to their students and the students' disabilities. In practice, students with VIs usually have other disabilities [8] [54], the adoption of these models will still highly rely on the help of TVIs.

I3Ms could lower the teaching load for TVIs, and TVIs should be highly involved into the design and implementation process of I3Ms to provide customization suggestions and domain knowledge.

I3Ms changed the way the TVIs interacted with their students. For example, T3 deliberately skipped some content in her lesson, allowing her student to learn this content by interacting with the model. All TVIs reported they had more time to observe their students as they interacted with the models. Based on these observations, they adjusted their teaching plans on the spot.

TVIs could help students benefit from the advantages of I3Ms and avoid potential challenges using their teaching techniques. For example, in the first study, the participants were dissatisfied with the limited sizes of the printed models. However, in the second study, the TVIs helped their students understand the scale of the model in relation to the referent object, and there was no feedback regarding model sizes.

\section{System Implications and Study Limitations}

Based on the feedback from the second study, Talkit++ could be further improved. First, the application could be more responsive by running GPU-accelerated algorithms. Second, 
we could deploy a skin color-based tracking method to avoid the need for stickers. Third, we should provide better audio cues to prevent students from moving models away. Also, the speech input could be more conversational, allowing students to ask the model questions about what it represents and its content.

In terms of limitations, In the first study, we only recorded the presentations for each group, and not their in-group discussions. Those in-group discussions could have provided deeper insights. In the second study, we did not let TVIs design models and add interactive elements by themselves. Instead, a researcher worked closely with the TVIs and implemented the designs himself. This approach is supported by the ADDIE model, which is widely adopted in practice. The I3Ms could be different, perhaps more effective, if they were entirely designed by TVIs who master the design tools.

\section{DESIGN GUIDELINES}

Based on the findings from both studies, we derived the following design guidelines. These aspirational guidelines will enable future model designers (e.g., TVIs, braillists) to create better I3Ms, and inform investigators about future research initiatives in digital fabrication and accessible educational technology.

\section{I3Ms should have effective tactile features}

- Use different printing materials to make the texture of a model similar to its referent object.

- Adjust the printing settings (e.g., speed, temperature, resolution) to avoid printing imperfections that may be confused for tactile textures or model components.

- Use tactile patterns to make the different components of a model more distinct from one another.

- Avoid adding redundant tactile features to a model, keep tactile information simple, clear, and salient.

- Use removable components to allow users to explore the relationships between a model's different components.

\section{I3Ms should contain both visual and auditory content}

- Use multi-level information to indicate the name of an element and its detailed description.

- Add audio effects and animations to provide additional information for students.

- For low vision students, use visual cues that direct their attention to salient parts of a model and high contrast colors to help them distinguish between model components.

\section{I3Ms should consider pedagogical methods}

- Provide an overview of an I3M before providing details.
- Adjust the content of a model according to a student's progress.

\section{FUTURE WORK}

We will continue to partner with TVIs to design I3M-based curricula. For example, we could use I3Ms to supplement instruction within a series of mathematics classes. We will also design a 3D modification tool for TVIs. The tool should allow them to easily modify and create models using online resources.

\section{CONCLUSION}

We conducted two studies to understand how to design effective I3Ms. In the first study, we led two design workshops with 35 TVIs, who modified sample models and added interactive elements to them. In the second study, we worked with three TVIs to design three I3Ms in an iterative instructional design process, and the TVIs used the I3Ms to teach their students at the end of this process. The two studies contributed to design guidelines for designing I3Ms, an updated mobile application to support the use of I3Ms in classrooms, and three sample I3Ms.

\section{ACKNOWLEDGMENTS}

This research was supported in part by Verizon Media Group and the National Science Foundation (NSF) under grant No. IIS-1746123. We thank all participants and their students for their time and helpful feedback. We also thank Jeannine Hobbes.

\section{REFERENCES}

[1] AER. 2018. Association for Education and Rehabilitation of the Blind and Visually Impaired. Retrieved January 4, 2019 from https://aerbvi. org/

[2] American Printing House. 2018. Product: DNA Twist. Retrieved January 4, 2019 from https://shop.aph.org/webapp/wcs/stores/servlet/ Product_DNATwist_1-08978-00P_10001_11051

[3] American Printing House. 2018. Product: Tactile World Globe. Retrieved January 4, 2019 from http://shop.aph.org/webapp/wcs/stores/ servlet/Product_Tactile\%20World\%20Globe_39214108P_10001_11051

[4] Apple. [n. d.]. AVAudioPlayer - AVFoundation | Apple Developer Documentation. Retrieved January 4, 2019 from https://developer.apple. com/documentation/avfoundation/avaudioplayer?language $=$ objc

[5] Apple. [n. d.]. AVSpeechSynthesizer - AVFoundation | Apple Developer Documentation. Retrieved January 4, 2019 from https://developer.apple.com/documentation/avfoundation/ avspeechsynthesizer?language $=$ objc

[6] Apple. [n. d.]. SFSpeechRecognizer - Speech | Apple Developer Documentation. Retrieved January 4, 2019 from https://developer.apple. com/documentation/speech/sfspeechrecognizer?language $=$ objc

[7] Apple. [n. d.]. UIImage - UIKit | Apple Developer Documentation. Retrieved January 4, 2019 from https://developer.apple.com/ documentation/uikit/uiimage?language $=$ objc

[8] Mark L. Batshaw. 2002. Children with disabilities. MacLennan \& Petty. 
[9] Quentin Bonnard, Severin Lemaignan, Guillaume Zufferey, Andrea Mazzei, Sebastien Cuendet, Nan Li, Ayberk Ozgur, and Pierre Dillenbourg. 2013. Chilitags 2: Robust Fiducial Markers for Augmented Reality and Robotics.

[10] Gary R. Bradski and Adrian. Kaehler. 2008. Learning OpenCV : computer vision with the OpenCV library. O'Reilly. 555 pages.

[11] Craig Brown and Amy Hurst. 2012. VizTouch: automatically generated tactile visualizations of coordinate spaces. In Proceedings of the Sixth International Conference on Tangible, Embedded and Embodied Interaction (TEI '12). ACM Press, New York, New York, USA, 131-138. https://doi.org/10.1145/2148131.2148160

[12] Emeline Brule, Gilles Bailly, Anke Brock, Frédéric Valentin, Grégoire Denis, and Christophe Jouffrais. 2016. MapSense: Multi-Sensory Interactive Maps for Children Living with Visual Impairments. In Proceedings of the 2016 CHI Conference on Human Factors in Computing Systems (CHI '16). 445-457. https://doi.org/10.1145/2858036.2858375

[13] Erin Buehler, Stacy Branham, Abdullah Ali, Jeremy J Chang, Megan Kelly Hofmann, Amy Hurst, and Shaun K. Kane. 2015. Sharing is Caring: Assistive Technology Designs on Thingiverse. In Proceedings of the 33rd Annual ACM Conference on Human Factors in Computing Systems (CHI '15). 525-534. https://doi.org/10.1145/2702123.2702525

[14] Erin Buehler, Niara Comrie, Megan Hofmann, Samantha McDonald, and Amy Hurst. 2016. Investigating the Implications of 3D Printing in Special Education. ACM Transactions on Accessible Computing 8, 3 (2016), 1-28. https://doi.org/10.1145/2870640

[15] Erin Buehler, Amy Hurst, and Megan Hofmann. 2014. Coming to grips: 3D printing for accessibility. In Proceedings of the 16th International ACM SIGACCESS Conference on Computers \& Accessibility (ASSETS '14). ACM Press, New York, New York, USA, 291-292. https://doi.org/ 10.1145/2661334.2661345

[16] Erin Buehler, Shaun K. Kane, and Amy Hurst. 2014. ABC and 3D: opportunities and obstacles to 3D printing in special education environments. In Proceedings of the 16th International ACM SIGACCESS Conference on Computers \& Accessibility (ASSETS '14). ACM Press, New York, New York, USA, 107-114. https://doi.org/10.1145/2661334.2661365

[17] Stéphanie Giraud, Anke M. Brock, Marc J-M Macé, and Christophe Jouffrais. 2017. Map Learning with a 3D Printed Interactive SmallScale Model: Improvement of Space and Text Memorization in Visually Impaired Students. Frontiers in Psychology 8 (6 2017), 930. https: //doi.org/10.3389/fpsyg.2017.00930

[18] Timo Götzelmann. 2016. LucentMaps: 3D Printed Audiovisual Tactile Maps for Blind and Visually Impaired People. In Proceedings of the 18th International ACM SIGACCESS Conference on Computers and Accessibility (ASSETS '16). ACM Press, New York, New York, USA, 81-90. https://doi.org/10.1145/2982142.2982163

[19] Timo Götzelmann. 2018. Visually Augmented Audio-Tactile Graphics for Visually Impaired People. ACM Transactions on Accessible Computing 11, 8 (2018), 31. https://doi.org/10.1145/3186894

[20] Timo Götzelmann and Aleksander Pavkovic. 2014. Towards automatically generated tactile detail maps by 3D printers for blind persons. In International Conference on Computers for Handicapped Persons, Vol. 8548 LNCS. 1-7. https://doi.org/10.1007/978-3-319-08599-9\{_\}1

[21] Noreen Grice, Carol Christian, Antonella Nota, and Perry Greenfield. 2015. 3D Printing Technology: A Unique Way of Making Hubble Space Telescope Images Accessible to Non-Visual Learners. Fournal of Blindness Innovation and Research 5, 1 (2015). https://doi.org/10.5241/ 5-66

[22] Jaume Gual, Marina Puyuelo, and Joaquim Lloveras. 2011. Universal design and visual impairment: Tactile products for heritage access. In Proceedings of the 18th International Conference on Engineering Design (ICED 11), Impacting Society Through Engineering Design, Vol. 5. 155-164. http://www.scopus.com/inward/record.url?eid=2-s2.
0-84858852744\&partnerID=tZOtx3y 1

[23] Jaume Gual, Marina Puyuelo, and Joaquim Lloveras. 2014. Threedimensional tactile symbols produced by 3D Printing: Improving the process of memorizing a tactile map key. British fournal of Visual Impairment 32, 3 (2014), 263-278. https://doi.org/10.1177/ 0264619614540291

[24] Jaume Gual, Marina Puyuelo, Joaquim Lloveras, and Lola Merino. 2012. Visual Impairment and urban orientation. Pilot study with tactile maps produced through 3D Printing. Psyecology 3, 2 (2012), 239-250. https://doi.org/10.1174/217119712800337747

[25] Anhong Guo, Jeeeun Kim, Xiang 'Anthony Chen, Tom Yeh, Scott E. Hudson, Jennifer Mankoff, and Jeffrey P. Bigham. 2017. Facade: Autogenerating Tactile Interfaces to Appliances. In Proceedings of the 2017 CHI Conference on Human Factors in Computing Systems (CHI '17). 5826-5838. https://doi.org/10.1145/3025453.3025845

[26] Megan Hofmann, Jeffrey Harris, Scott E. Hudson, and Jennifer Mankoff. 2016. Helping Hands: Requirements for a Prototyping Methodology for Upper-limb Prosthetics Users. In Proceedings of the SIGCHI Conference on Human Factors in Computing Systems (CHI '16). ACM Press, New York, New York, USA, 1769-1780. https://doi.org/10.1145/2858036. 2858340

[27] Leona Holloway, Kim Marriott, and Matthew Butler. 2018. Accessible Maps for the Blind : Comparing 3D Printed Models with Tactile Graphics. In Proceedings of the 2018 CHI Conference on Human Factors in Computing Systems (CHI '18). ACM Press, New York, New York, USA, Paper 198, 13 pages. https://doi.org/10.1145/3173574.3173772

[28] Michele Hu. 2015. Exploring New Paradigms for Accessible 3D Printed Graphs. In Proceedings of the 17th International ACM SIGACCESS Conference on Computers \& Accessibility (ASSETS '15). 365-366. https://doi.org/10.1145/2700648.2811330

[29] Amy Hurst and Jasmine Tobias. 2011. Empowering individuals with do-it-yourself assistive technology. In The proceedings of the 13th international ACM SIGACCESS conference on Computers and accessibility (ASSETS '11). 11-18. https://doi.org/10.1145/2049536.2049541

[30] Shaun K. Kane and Jeffrey P. Bigham. 2014. Tracking@ stemxcomet: teaching programming to blind students via 3D printing, crisis management, and twitter. In Proceedings of the 45th ACM technical symposium on Computer science education (SIGCSE '14). ACM Press, New York, New York, USA, 247-252. https://doi.org/10.1145/2538862.2538975

[31] Jeeeun Kim and Tom Yeh. 2015. Toward 3D-Printed Movable Tactile Pictures for Children with Visual Impairments. In Proceedings of the 33rd Annual ACM Conference on Human Factors in Computing Systems (CHI '15). 2815-2824. https://doi.org/10.1145/2702123.2702144

[32] Michael A. Kolitsky. 2014. 3D Printed Tactile Learning Objects: Proof of Concept. Fournal of Blindness Innovation and Research 4, 1 (10 2014), $4-51$.

[33] Michael A. Kolitsky. 2014. Making Tactile 3D Prints Talk. Retrieved January 4, 2019 from http://www.instructables.com/id/ Making-Tactile-3D-Prints-Talk/

[34] Samantha McDonald, Joshua Dutterer, Ali Abdolrahmani, Shaun K. Kane, and Amy Hurst. 2014. Tactile aids for visually impaired graphical design education. In Proceedings of the 16th International ACM SIGACCESS Conference on Computers \& Accessibility (ASSETS '14). ACM Press, New York, New York, USA, 275-276. https://doi.org/10.1145/2661334. 2661392

[35] M. David Merrill, Leston Drake, Mark J. Lacy, and Jean Pratt. 1996. Reclaiming instructional design. Educational Technology 36, 5 (1996), 5-7.

[36] Huaishu Peng, François Guimbretière, James McCann, and Scott Hudson. 2016. A 3D Printer for Interactive Electromagnetic Devices. In Proceedings of the 29th Annual Symposium on User Interface Software and Technology (UIST '16). 553-562. https://doi.org/10.1145/2984511. 
2984523

[37] Huaishu Peng, Jennifer Mankoff, Scott E. Hudson, and James McCann. 2015. A Layered Fabric 3D Printer for Soft Interactive Objects. In Proceedings of the 33rd Annual ACM Conference on Human Factors in Computing Systems (CHI '15). 1789-1798. https://doi.org/10.1145/ 2702123.2702327

[38] George M. Piskurich. 2015. Rapid instructional design: Learning ID fast and right. John Wiley \& Sons.

[39] Andreas Reichinger, Anton Fuhrmann, Stefan Maierhofer, and Werner Purgathofer. 2016. Gesture-Based Interactive Audio Guide on Tactile Reliefs. In Proceedings of the 18th International ACM SIGACCESS Conference on Computers and Accessibility (ASSETS '16). ACM Press, New York, New York, USA, 91-100. https://doi.org/10.1145/2982142.2982176

[40] Robert A. Resier. 2002. A history of Instructional Design and Technology. In Trends and Issues in Instructional Design. 26-53.

[41] Audrey C. Rule, Greg P. Stefanich, Robert M. Boody, and Belinda Peiffer. 2011. Impact of adaptive materials on teachers and their students with visual impairments in secondary science and mathematics classes. International fournal of Science Education 33, 6 (2011), 865-887. https: //doi.org/10.1080/09500693.2010.506619

[42] Johnny Saldana. 2015. The Coding Manual for Qualitative Researchers. Vol. 109. Sage. https://doi.org/10.1017/CBO9781107415324.004

[43] Martin Schmitz, Mohammadreza Khalilbeigi, Matthias Balwierz, Roman Lissermann, Max Mühlhäuser, and Jürgen Steimle. 2015. Capricate: A Fabrication Pipeline to Design and 3D Print Capacitive Touch Sensors for Interactive Objects. Proceedings of the 28th Annual ACM Symposium on User Interface Software \& Technology (UIST '15) (2015), 253-258. https://doi.org/10.1145/2807442.2807503

[44] Lei Shi. 2015. Talkabel: A Labeling Method for 3D Printed Models. In Proceedings of the 17th International ACM SIGACCESS Conference on Computers \& Accessibility (ASSETS'15). ACM, New York, New York, USA, 361-362. https://doi.org/10.1145/2700648.2811327

[45] Lei Shi, Ross McLachlan, Yuhang Zhao, and Shiri Azenkot. 2016. Magic Touch: Interacting with 3D Printed Graphics. In Proceedings of the 18th International ACM SIGACCESS Conference on Computers and Accessibility (ASSETS '16). ACM Press, New York, New York, USA, 329-330. https://doi.org/10.1145/2982142.2982153

[46] Lei Shi, Idan Zelzer, Catherine Feng, and Shiri Azenkot. 2016. Tickers and Talker: An Accessible Labeling Toolkit for 3D Printed Models. In Proceedings of the 2016 CHI Conference on Human Factors in Computing Systems (CHI '16). ACM Press, New York, New York, USA, 4896-4907. https://doi.org/10.1145/2858036.2858507

[47] Lei Shi, Yuhang Zhao, and Shiri Azenkot. 2017. Designing Interactions for 3D Printed Models with Blind People. In In Proceedings of the 19th International ACM SIGACCESS Conference on Computers \& Accessibility (ASSETS '17). https://doi.org/10.1145/3132525.3132549
[48] Lei Shi, Yuhang Zhao, and Shiri Azenkot. 2017. Markit and Talkit: A Low-Barrier Toolkit to Augment 3D Printed Models with Audio Annotations. In Proceedings of the 30th Annual ACM Symposium on User Interface Software and Technology (UIST '17). ACM Press, New York, New York, USA, 493-506. https://doi.org/10.1145/3126594.3126650

[49] Abigale Stangl, Chia-Lo Hsu, and Tom Yeh. 2015. Transcribing across the senses: community efforts to create 3D printable accessible tactile pictures for young children with visual impairments. In Proceedings of the 17th International ACM SIGACCESS Conference on Computers $\&$ Accessibility (ASSETS '15). ACM Press, New York, New York, USA, 127-137. https://doi.org/10.1145/2700648.2809854

[50] Abigale Stangl, Jeeeun Kim, and Tom Yeh. 2014. 3D printed tactile picture books for children with visual impairments. In Proceedings of the 2014 conference on Interaction design and children (IDC '14). ACM Press, New York, New York, USA, 321-324. https://doi.org/10.1145/ 2593968.2610482

[51] Brandon Taylor, Anind Dey, Dan Siewiorek, and Asim Smailagic. 2016. Customizable 3D Printed Tactile Maps as Interactive Overlays. In ASSETS '16. ACM Press, New York, New York, USA, 71-79. https: //doi.org/10.1145/2982142.2982167

[52] Brandon T. Taylor, Anind K. Dey, Dan P. Siewiorek, and Asim Smailagic. 2015. TactileMaps.net: A web interface for generating customized 3Dprintable tactile maps. In Proceedings of the 17th International ACM SIGACCESS Conference on Computers \& Accessibility (ASSETS '15). 427428. https://doi.org/10.1145/2700648.2811336

[53] Jolene K. Teske, Phyllis Gray, Mason A. Kuhn, Courtney K. Clausen, Latisha L. Smith, Sukainah A. Alsubia, Maryam Ghayoorad, Audrey C. Rule, and Jean Suchsland Schneider. 2014. Teacher-Made Tactile Science Materials with Critical and Creative Thinking Activities for Learners Including Those with Visual Impairments. Online Submission (2014). https://doi.org/10.1186/1752-0509-2-75

[54] Martha Thurlow, Christopher Johnstone, Joseph Timmons, and Jason Altman. 2009. Survey of teachers of students with visual impairments: Students served and their access to state assessments of reading. Minneapolis, MN: University of Minnesota, Technology Assisted Reading Assessment (2009).

[55] Henry B. Wedler, Sarah R. Cohen, Rebecca L. Davis, Jason G. Harrison, Matthew R. Siebert, Dan Willenbring, Christian S. Hamann, Jared T. Shaw, and Dean J. Tantillo. 2012. Applied Computational Chemistry for the Blind and Visually Impaired. Fournal of Chemical Education 89, 11 (2012), 1400-1404. https://doi.org/Doi10.1021/Ed3000364

[56] Yang Zhang, Gierad Laput, and Chris Harrison. 2017. Electrick : Low Cost Touch Sensing Using Electric Field Tomography. In Proceedings of the 2017 CHI Conference on Human Factors in Computing Systems (CHI '17) (2017), 1-14. https://doi.org/10.1145/3025453.3025842 